\title{
VARIATION NORM CONVERGENCE OF FUNCTION SEQUENCES ${ }^{1}$
}

\author{
RANDOLPH CONSTANTINE, JR.
}

ABSTRACT. We prove that a pointwise convergent sequence of convex functions with a continuous limit converges with respect to the total variation norm. This yields a theorem on convexity-preserving operators which has as a corollary the result that a complex function $f$ is absolutely continuous on $[0,1]$ if and only if the sequence $B .(f)$ of Bernstein polynomials of $f$ converges to $f$ with respect to the total variation norm.

In this paper a theorem which is analogous to Dini's theorem is proved;

Theorem 1. If $f$. is a pointwise convergent sequence of real-valued functions, each of which is convex on $[a, b]$ and the limit function $F$ is continuous on $[a, b]$, then the sequence $f$. converges to $F$ with respect to the total variation norm on $[a, b]$.

This is then used to prove

Theorem 2. Suppose $T$. is a sequence of linear operators from $A C[a, b]$ into $A C[a, b]$ such that for each $f \in A C[a, b],(1) T .(f)$ converges pointwise to $f$ on $[a, b]$; (2) if $f$ is convex on $[a, b]$ and $n$ is a nonnegative integer, $T_{n}(f)$ is convex on $[a, b]$; and (3) there is a number $M \geq 0$ such that for each nonnegative integer $n, \int_{a}^{b}\left|d\left(T_{n}(f)\right)\right| \leq M \int_{a}^{b}|d f|$. Then, for each $f \in A C[a, b]$, the function sequence $T .(f)$ converges to $f$ with respect to the total variation norm.

Corollary. A complex-valued function $f$ is absolutely continuous on

Presented to the Society, January 25, 1973; received by the editors March 10, 1973 and, in revised form, October 22, 1973.

AMS (MOS) subject classifications (1970). Primary 41A30, 41A25; Secondary 26A51.

Key words and phrases. Total variation norm, convex function, absolutely continuous function, Bernstein polynomial.

1 The results of this paper constitute a portion of the author's dissertation which was written under the direction of J. S. Mac Nerney at the University of Houston. 
$[0,1]$ if and only if the sequence B.f of Bernstein polynomials of $f$ converges to $f$ with respect to the total variation norm.

An example is given to show that Theorem 1 does not extend to differences of convex functions.

Definitions and notation. A real-valued function $f$ on $[a, b]$ is said to be convex on $[a, b]$ (or simply, convex) provided that for each $[u, v] \subseteq[a, b]$ and each number $t, 0<t<1$,

$$
f((1-t) u+t v) \geq(1-t) f(u)+t f(v) .
$$

I denotes the identity function on the complex plane, and we employ the convention that $l^{0}$ is the constant function 1 so that for each nonnegative integer $n$ and each complex number $x, I^{n}(x)=x^{n}$. Hence if $f$ is a complex function on $[0,1]$, the Bernstein polynomial sequence of $f$ is defined by

$$
B_{0} f=f(0) \text { and } B_{n} f=\sum_{p=0}^{n}\left(\begin{array}{l}
n \\
p
\end{array}\right) f\left(\frac{p}{n}\right) I^{p}(1-I)^{n-p}
$$

for $n$-a positive integer. For a complex function $f$ from a subset of the real numbers, $f\left(x_{-}\right)$and $f(x+)$ respectively denote the left and right hand limits of $f$ at $x$ in case the limit exists; if $S$ is a subset of the domain of $f$ and $f(S)$ is a bounded set, $|f|_{S}=\sup \{|f(x)|: x$ in $S\}$; if $f$ is of bounded variation on $[a, b], \int_{a}^{b}|d f|$ denotes the total variation of $f$ on $[a, b]$. The notation $] a, b$ [ denotes the open interval $\{x: a<x<b\}$ and $(a, b)$ is reserved for an ordered pair.

1. Convex functions. We note without proof the following properties of convex functions:

If $f$ is a convex function on $[a, b]$, then

(1) $f$ is continuous on $] a, b[$;

(2) each of $f(a+)$ and $f(b-)$ exists and $f(a) \leq f(a+)$ and $f(b-) \geq f(b)$;

(3) if, in addition, $f$ is nonconstant on $] a, b[$, then only one of the following statements is true:

(a) $f$ is nondecreasing on $[a, b[$,

(b) $f$ is nonincreasing on $] a, b]$,

(c) there is a number $x_{0}$ in $] a, b$ [ such that $f$ is nondecreasing on $\left[a, x_{0}\right], f$ is nonincreasing on $\left[x_{0}, b\right]$ and $f$ is nonconstant on $] a, x_{0}[$ and on $] x_{0}, b[$;

(4) if in addition $f$ is continuous at $a$ and at $b$, then $f$ is absolutely continuous on $[a, b]$; 
(5) a continuous polygonal function is a difference of continuous convex polygonal functions.

Theorem 0 . If $f$. is a pointwise convergent sequence of convex functions on $[a, b]$ and $F$ denotes the limit function, then

(1) $F$ is convex on $[a, b]$ and

(2) if $F$ is continuous on $[a, b]$, then $f$. converges uniformly on $[a, b]$.

Proof. Part (1) follows from the facts that a pointwise convergent function sequence converges uniformly on a finite set, and, hence, for each $[u, v]$ $C[a, b]$ and $t, 0<t<1, F((1-t) u+t v) \geq(1-t) F(u)+t F(v)$ must be true since $f_{n}((1-t) u+t v) \geq(1-t) f_{n}(u)+t f_{n}(v)$ for each $n$.

Proof of (2). There is an $x_{0} \in[a, b]$ such that $F$ is monotone on each of $\left[a, x_{0}\right]$ and $\left[x_{0}, b\right]$. Hence it is enough to prove the theorem under the added assumption that $F$ is nondecreasing. Suppose $c>0$. There is an increasing sequence $\left\{t_{i}\right\}_{0}^{k}$ with $t_{0}=a$ and $t_{k}=b$ such that $F\left(t_{i}\right)-F\left(t_{i-1}\right)<$ $c, i=1, \cdots, k$.

Let $s_{i}=\left(t_{i-1}+t_{i}\right) / 2, i=1, \cdots, k$. If $t_{i-1} \leq x \leq s_{i}$ then, since $f_{n .}$ is convex,

$$
\left(f_{n}\left(s_{i}\right)-f_{n}(x)\right) /\left(s_{i}-x\right) \geq\left(f_{n}\left(t_{i}\right)-f_{n}\left(s_{i}\right)\right) /\left(t_{i}-s_{i}\right) .
$$

Since $\left(s_{i}-x\right) /\left(t_{i}-s_{i}\right) \leq\left(s_{i}-t_{i-1}\right) /\left(t_{i}-s_{i}\right)=1$, this implies

$$
f_{n}(x) \leq f_{n}\left(s_{i}\right)+\left|f_{n}\left(t_{i}\right)-f_{n}\left(s_{i}\right)\right|
$$

and hence

$$
\sup _{t_{i-1} \leq x \leq s}\left\{f_{n}(x)-F(x)\right\} \leq f_{n}\left(s_{i}\right)-F\left(t_{i-1}\right)+\left|f_{n}\left(t_{i}\right)-f_{n}\left(s_{i}\right)\right| .
$$

Similarly, if $s_{i}<x \leq t_{i}$ then

$$
\left(f_{n}(x)-f_{n}\left(s_{i}\right)\right) /\left(x-s_{i}\right) \leq\left(f_{n}\left(s_{i}\right)-f_{n}\left(t_{i-1}\right)\right) /\left(s_{i}-t_{i-1}\right),
$$

whence

(B)

$$
\sup _{s_{i} \leq x \leq t_{i}}\left\{f_{n}(x)-F(x)\right\} \leq f_{n}\left(s_{i}\right)-F\left(x_{i}\right)+\left|f_{n}\left(s_{i}\right)-f_{n}\left(t_{i-1}\right)\right| .
$$

Also, if $t_{i-1} \leq x \leq t_{i}$, then $f_{n}(x) \geq \min \left\{f_{n}\left(t_{i-1}\right), f_{n}\left(t_{i}\right)\right\}$, and

$$
\sup _{t_{i-1} \leq x \leq t_{i}}\left\{F(x)-f_{n}(x)\right\} \leq F\left(t_{i}\right)-\min \left\{f_{n}\left(t_{i-1}\right), f_{n}\left(t_{i}\right)\right\} \text {. }
$$

As $n \rightarrow \infty$, the right-hand side of each of (A), (B) and (C) has a limit less than $c$, and the theorem is proved. 
Lemma 1.1. If $f$ is convex on $[a, b], e>0, g$ is convex on $[a, b]$ such that

$$
|f-g|_{[a, b]} \leq e \quad \text { and } \quad P=\frac{f(b)-f(a)}{b-a}(I-a)+f(a)
$$

then

$$
\int_{a}^{b}|d(f-P)|=2|f-P|[a, b]
$$

and

$$
\int_{a}^{b}|d(g-P)| \leq \int_{a}^{b}|d(f-P)|+4 e
$$

Proof. (1) follows immediately from the unproved assertion (3) about convex functions. To prove (2) we note that $g-P$ is convex and apply the same assertion (3) in the three separate cases. Let us consider only the case where there exists a number $x_{0}$ in $] a, b$ [ such that $g-P$ is nondecreasing on $\left[a, x_{0}\right]$, nonincreasing on $\left[x_{0}, b\right]$ and nonconstant on each of $] a, x_{0}[$ and $] x_{0}, b[$. Thus

$$
\begin{aligned}
\int_{a}^{b}|d(g-P)| & =2(g-P)\left(x_{0}\right)-(g-P)(a)-(g-P)(b) \\
& =2 g\left(x_{0}\right)-2 P\left(x_{0}\right)-g(a)+f(a)-g(b)+f(b) \\
& \leq 2\left\{f\left(x_{0}\right)+e\right\}-2 P\left(x_{0}\right)+e+e \\
& =2\left\{f\left(x_{0}\right)-P\left(x_{0}\right)\right\}+4 e \leq 2|f-P|[a, b]+4 e \\
& =\int_{a}^{b}|d(f-P)|+4 e .
\end{aligned}
$$

We omit proof of the other two cases.

Lemma 1.2, which follows, was proved independently by the author for convex functions only. It follows immediately from a result of J. R. Edwards and S. G. Wayment [1, p. 254] on absolutely continuous functions and the fact that a continuous convex function is absolutely continuous.

Lemma 1.2. If $F$ is a continuous convex function on $[a, b]$ and $c>0$, then there is an increasing sequence $\left\{t_{p}\right\}_{0}^{n}$ with $t_{0}=a$ and $t_{n}=b$ such that if $P$ is the function on $[a, b]$ defined by

$$
P(x)=\frac{F\left(t_{p+1}\right)-F\left(t_{p}\right)}{t_{p+1}-t_{p}}\left(x-t_{p}\right)+F\left(t_{p}\right) \text { for } x \text { in }\left[t_{p}, t_{p+1}\right],
$$

then $\int_{a}^{b}|d(F-P)|<c$.

Theorem 1. If $f$. is a pointwise convergent sequence of functions on 
$[a, b]$ each of which is convex on $[a, b]$ and the limit function $F$ is continuous on $[a, b]$, then the sequence $f$. converges to $F$ in the total variation norm on $[a, b]$.

Proof. Suppose the hypothesis and let $c>0$. There is an increasing sequence $\left\{t_{p}\right\}_{0}^{n}$ with $t_{0}=a$ and $t_{n}=b$ such that if $P$ is the function as defined in Lemma 1.2 for $F$, then $\int_{a}^{b}|d(F-P)|<c / 4$. Let $e=c /(8 n)$. There is a positive integer $N$ such that if $q$ is an integer, $q>N$, then $\left|f_{q}-F\right|_{[a, b]}$ $<e$ by Theorem 0 . For each integer $q>N$

$$
\begin{aligned}
\int_{a}^{b}\left|d\left(f_{q}-F\right)\right| & \leq \int_{a}^{b}\left|d\left(f_{q}-P\right)\right|+\int_{a}^{b}|d(P-F)| \\
& <\sum_{p=0}^{n-1} \int_{t_{p}}^{t} p+1\left|d\left(f_{q}-P\right)\right|+\frac{c}{4} .
\end{aligned}
$$

But Lemma 1.1 and the fact that $\left|f_{q}-F\right|_{\left[t_{p}, t_{p+1}\right]} \leq\left|f_{q}-F\right|_{[a, b]}<e$ imply that for each integer $p, 0 \leq p \leq n-1$,

$$
\int_{t_{p}}^{t} p+1\left|d\left(f_{q}-P\right)\right| \leq \int_{t_{p}}^{t} p+1\left|d\left(F-f_{q}\right)\right|+4 e .
$$

Whence we see that

$$
\begin{aligned}
\sum_{p=0}^{n-1} \int_{t_{p}}^{t} p+1\left|d\left(f_{q}-P\right)\right| & \leq \sum_{p=0}^{n-1}\left\{\int_{t_{p}}^{t} p+1|d(F-P)|+4 e\right\} \\
& =\int_{a}^{b}|d(F-P)|+4 n e=\int_{a}^{b}|d(f-P)|+\frac{c}{2}<\frac{3 c}{4} .
\end{aligned}
$$

Thus $\int_{a}^{b}\left|d\left(f_{q}-F\right)\right|<c$ for each integer $q>N$.

Corollary. If $F$ is a continuous convex function on $[0,1]$, then the sequence B.F of Bermstein polynomials of $F$ converges to $F$ with respect to the total variation norm on $[0,1]$.

Proof. This is an immediate consequence of the well-known facts that since $F$ is continuous, $B . F$ converges uniformly to $F$ and that for each nonnegative integer, $n, B_{n} F$ is convex on $[0,1]$; cf. Lorentz $[3$, p. 5 and p. 23 resp.].

Remark. Theorem 1 does not extend to sequences of differences of convex functions, as may be seen from the following example: let $f$. be a sequence of functions on $[0,1]$ such that for each positive integer $n$, and nonnegative integer $p<2^{n}$ 


$$
f_{n}(x)= \begin{cases}x-p / 2^{n} & \text { if } x \in\left[p / 2^{n},(p+1) / 2^{n}[\text { and } p \text { is even, }\right. \\ 1 / 2^{n}-\left(x-p / 2^{n}\right) & \text { if } x \in\left[p / 2^{n},(p+1) / 2^{n}\right] \text { and } p \text { is odd. }\end{cases}
$$

Each function $f_{n}$ is a continuous polygonal function with $\int_{a}^{b}\left|d f_{n}\right|=1$, and the sequence $f$. converges uniformly to the constant function 0 .

2. Absolutely continuous functions. A function $f$ is said to be absolutely continuous on $[a, b]$ provided that for each $c>0$ there is a positive number $d$ such that if $\left\{\left[u_{p}, v_{p}\right]\right\}_{0}^{n}$ is a sequence of nonoverlapping subintervals of $[a, b]$ with $\sum_{p=0}^{n}\left(v_{p}-u_{p}\right)<d$, then $\sum_{p=0}^{n}\left|f\left(v_{p}\right)-f\left(u_{p}\right)\right|<c$. It is well known [1] that the class $A C[a, b]$ of all absolutely continuous real-valued functions on $[a, b]$ is complete with respect to the total variation norm and that the polygonal functions form a dense subset thereof.

Theorem 2. Suppose $T$. is a sequence of linear operators from $A C[a, b]$ into $A C[a, b]$ such that for each $f$ in $A C[a, b],(1) T .(f)$ converges pointwise to $f$ on $[a, b] ;$; (2) if $f$ is convex on $[a, b]$ and $n$ is a nonnegative integer, $T_{n}(f)$ is convex on $[a, b]$; and (3) there is a number $M \geq 0$ such that for each nonnegative in. teger $n, \int_{a}^{b}\left|d\left(T{ }_{n}(f)\right)\right| \leq M \int_{a}^{b}|d f|$. Then, for each $f \in A C[a, b]$, the function sequence $T .(f)$ converges to $f$ with respect to the total variation norm.

Proof. Let $B$ denote the set of all real-valued functions $f$ on $[a, b]$ such that $T$. $f$ converges to $f$ with respect to the total variation norm. $B$ is closed with respect to the total variation norm, for if $F$ is the limit with respect to the total variation norm of a sequence $f$. with values in $\mathcal{B}$, then

$$
\begin{aligned}
\int_{a}^{b}\left|d\left(F-T_{n} F\right)\right| \leq & \int_{a}^{b}\left|d\left(F-f_{k}\right)\right|+\int_{a}^{b}\left|d\left(f_{k}-T_{n}\left(f_{k}\right)\right)\right| \\
& +\int_{a}^{b}\left|d\left(T_{n}\left(f_{k}\right)-T_{n}(F)\right)\right| .
\end{aligned}
$$

But from part (3) of the hypothesis we have that

$$
\int_{a}^{b}\left|d\left(T_{n}\left(f_{k}\right)-T_{n}(F)\right)\right|=\int_{a}^{b}\left|d\left(T_{n}\left(f_{k}-F\right)\right)\right| \leq M \int_{a}^{b}\left|d\left(f_{k}-F\right)\right| .
$$

Thus

$$
\int_{a}^{b}\left|d\left(F-T_{n}(F)\right)\right| \leq(M+1) \int_{a}^{b}\left|d\left(F-f_{k}\right)\right|+\int_{a}^{b}\left|d\left(f_{k}-T_{n}\left(f_{k}\right)\right)\right|,
$$

from which it is clear that $B$ is closed with respect to the total variation norm.

If $P$ is a polygonal function on $[a, b]$ then $P$ is a difference of continuous convex functions, say $P=h-k$. But for each nonnegative integer $n$, 
$T_{n} P=T_{n}(h-k)=T_{n}(h)-T_{n}(k)$; whence by Theorem 1 and parts (1) and (2) of the hypothesis, $P$ must belong to $\mathscr{B}$. Thus $\mathbb{B}=A C[a, b]$ since $\mathscr{B}$ contains all polygonal functions and is closed with respect to the total variation norm.

Corollary. A complex-valued function $f$ is absolutely continuous on $[0,1]$ if and only if the sequence B.f of Bernstein polynomials of $f$ converges to $f$ with respect to the total variation norm.

Proof. Let us note that if $f$ is a complex-valued absolutely continuous function on $[0,1]$, then each of $\operatorname{Re} f$ and $\operatorname{Im} f$ is absolutely continuous; and if $n$ is a nonengative integer, $B_{n} f=B_{n} \operatorname{Re} f+i B_{n} \operatorname{Im} f$. Thus it is sufficient to suppose $f$ to be real valued, and we do so. Since any polynomial is absolutely continuous on $[0,1]$, then any function $f$ on $[0,1]$ such that $B . f$ converges to $f$ with respect to the total variation norm must perforce be absolutely continuous. Theorem 2 yields the converse.

Comment. The corollary to Theorem 2 has been obtained independently by G. G. Johnson, who used methods different from ours. While the results herein give no estimate on the size of $\int_{0}^{1}\left|d\left(F-B_{n}(F)\right)\right|$, they offer an extension of a result of W. Hoeffding [2, p. 349] that: If $f$ is a continuous convex function such that $\int_{0}^{1} I^{1 / 2}(1-I)^{1 / 2} d\left(f^{\prime}\right)$ exists, then $B . f$ converges with respect to the total variation norm. Some applications of these results to moment problems will appear in a subsequent paper.

Acknowledgment. I thank the referee for his helpful comments and suggestions; especially for indicating a way to significantly shorten the proof of Theorem 0 , and for suggesting that the proof of my original Theorem 2 might indeed be a proof of a stronger theorem.

\section{REF ERENCES}

1. J. R. Edwards and S. G. Wayment, Representations for transformations continuous in the BV norm, Trans. Amer. Math. Soc. 154 (1971), 251-265. MR 43 \#466.

2. Wassily Hoeffding, The $L_{1}$ norm of the approximation error for Bernstein-type polynomials, J. Approximation Theory 4 (1971), 347-356. MR 44 \#664.

3. G. G. Lorentz, Bernstein polynomials, Mathematical Expositions, no. 8, Univ. of Toronto Press, Toronto, 1953. MR 15, 217.

DEPARTMENT OF MATHEMATICAL SCIENCES, CLEMSON UNIVERSITY, CLEMSON, SOUTH CAROLINA 29631

Current address: Department of Mathematics, Erskine College, Due West, South Carolina 29639 